ORIGINAL ARTICLE

\title{
Neurotological status in Behçet's disease and its ophthalmological correlates
}

\author{
Y A Bayazıt, C Evereklioğlu, E Ozer, N Kırtak, N Bayazıt, M Kanlıkama, S İnalöz
}

Postgrad Med J 2004;80:724-728. doi: 10.1136/pgmj.2003.017756

See end of article for authors' affiliations

Correspondence to:

$\operatorname{Dr}$ Y A Bayazıt

Department of

Otolaryngology, Faculty of

Medicine, Gazi University,

Besevler, Ankara, Turkey;

bayazity@yahoo.com

Submitted

4 December 2003

Accepted

11 February 2004
Objective: To assess neurotological status and its ophthalmological correlates in Behçet's disease.

Study design: A cross sectional study in which 17 patients with Behçet's disease and 13 healthy controls were included.

Patients and methods: The patients were divided into two groups: those with and without end stage ocular involvement. Neurotological status was evaluated with audiological tests (pure tone and speech audiometry, tympanometry, short increment sensitivity index, tone decay, and evoked response audiometry), and Dix-Halpike positional testing.

Results: The positional test was normal. The audiological test results of the patients with and without end stage ocular involvement were not significantly different $(p>0.05)$. A sloping audiogram with bilateral symmetric and mild sensorineural hearing loss was the main audiogram obtained. There was no relationship between the presence or absence of the end stage ocular involvement and the otological parameters studied excluding I-III interval on evoked response audiometry ( $p>0.05)$.

Conclusion: Although the main underlying pathogenetic factor in Behçet's disease is the autoimmune vasculitis, the mechanisms involved in the pathogenesis of neurological and ocular damage may be different. Alternatively, the differential involvement of certain organ systems may not reflect the nature of the disease process itself, but rather the manner in which each organ responds to injury.
$\mathrm{D}$ r Hulusi Behçet, a Turkish physician from Istanbul, first described Behçet's disease in 1937 as a triad of oral aphthae and genital ulcerations with hypopion uveitis. ${ }^{1}$ The disease is a chronic inflammatory disorder involving the small vessels, which is of unknown aetiology, and is characterised by recurrent aphthous ulceration of the oral and pharyngeal mucous membranes and the genitalia, skin lesions, severe uveitis, retinal vasculitis, and optic atrophy. It has a worldwide distribution with a prevalence ranging from 1:1000-1:10 000 in Japan and Turkey to 1:500 000 in North America and Europe. ${ }^{2}$ Although the aetiopathogenesis remains obscure, Behçet's disease is considered to be autoimmune because of the common denominator of vasculitis in most patients. Circulating antibodies to human oral mucous membrane and immune complexes are found in about 50\% of cases. It appears to be linked to HLA-B5 and HLA-DR5 alloantigens. ${ }^{3}$ This autoimmune disease may be familial and has a genetic basis. ${ }^{4}$ It can also be considered multifactorial as genetic predisposition, abnormalities in both cellular and humoral immune system, and endothelial cell dysfunction are involved in its pathogenesis.

This unique disorder begins between the second and third decades of life (range 0-72 years) with a 3:2 male to female ratio. $^{2}$ It affects almost every tissue and organ in the body without exception including the joints, gastrointestinal system, ${ }^{3}$ cornea, ${ }^{6}$ kidney, ${ }^{7}$ and the lung. ${ }^{8}$ Although several aetiopathogenetic molecules including adrenomedullin, ${ }^{9}$ nitric oxide, ${ }^{10}$ proinflammatory cytokines, ${ }^{11}$ and malondialdehyde $^{12}$ have been implicated, the intermittent nature and the lack of consistent response to treatment make the underlying aetiology difficult to define. The perivasculitis is a characteristic feature in Behçet's disease, and systemic venous thrombosis is seen in $25 \%$ of the patients. ${ }^{13}$ Central nervous system involvement occurs in $10 \%$ to $25 \%$ of cases, of which the neurological manifestations are variable, severe, and progressive. ${ }^{2}$ All parts of neuraxis may be involved. ${ }^{14}$ In 1961, it was first proposed that Behçet's disease could involve the inner ear. ${ }^{15}$ The incidence of otological involvement ranges from $12 \%$ to $80 \% .{ }^{16-22}$

For this study, we assessed the neurotological status in patients with Behçet's disease and investigated whether it is associated or correlated with the most disastrous outcome, end stage ocular disease.

\section{SUBJECTS AND METHODS}

Informed consent was obtained from all participants. Patients with Behçet's disease were recruited from the departments of ophthalmology and dermatology at the research hospital in our university. A total of 17 consecutive patients who were diagnosed with Behçet's disease were enrolled in the study. Thirteen healthy subjects (mostly hospital staff) comprised the control group (three women and 10 men, mean age 29.6 years). The subjects in the control group were evaluated by history and physical examination. They did not have a history of chronic disease (for example, cardiovascular, metabolic, endocrine, rheumatoid, neurological or autoimmune disease), a history of chronic drug use, ototoxicity, acoustic trauma, or otological surgery.

Patients with Behçet's disease had to fulfil the criteria of the International Study Group for Behçet's Disease. ${ }^{23}$ There were no patients with neuro-Behçet on neurological examination and neuroimaging. Patients' details were obtained from case notes and ocular examinations were performed by an experienced ophthalmologist using a standard procedure. The diagnosis of uveitis was made according to the recommendations of the International Uveitis Study Group. ${ }^{24}$ In particular, a history of systemic thrombosis and evidence of retinal vascular occlusion were sought. Where the posterior segment could not be visualised, such patients were accepted as having end stage ocular disease (phthisical or completely

Abbreviations: ANOVA, analysis of variance; ERA, evoked response audiometry; PTA, pure tone average; SDS, speech discrimination score; SISI, short increment sensitivity index; SRT, speech reception threshold 
Table 1 Audiometric results in patients without (group 1) or with (group 2) end stage ocular involvement and healthy controls

\begin{tabular}{|c|c|c|c|c|c|c|c|c|}
\hline \multirow[b]{3}{*}{ Ear } & & & \multicolumn{4}{|c|}{ End stage ocular involvement } & & \\
\hline & \multicolumn{2}{|c|}{ Overall $(n=17)$} & \multicolumn{2}{|c|}{ Group $1(n=9)$} & \multicolumn{2}{|c|}{ Group $2(n=8)$} & \multicolumn{2}{|c|}{ Controls ( $n=13$ ) } \\
\hline & Right & Left & Right & Left & Right & Left & Right & Left \\
\hline PTA (dB) & 13.9 & 15.2 & 11.8 & 11.9 & 16.4 & 19 & 10.1 & 10.6 \\
\hline SRT (dB) & 13.5 & 15.3 & 11.1 & 12.2 & 16.3 & 18.8 & 11.4 & 12.9 \\
\hline SDS (\%) & 91.5 & 90.4 & 90.4 & 88.4 & 92.8 & 92.5 & 94 & 95.2 \\
\hline
\end{tabular}

blind). The patients did not have another disease like acoustic trauma, Meniere's disease, ototoxicity, or otitis media that could impact on the neurotological assessments. Behçet's patients were divided into two groups: those without (group 1 ) and those with (group 2) end stage ocular involvement.

A history was taken, and otolaryngological examination was performed. Dix-Halpike positional testing evaluated positional vertigo. Audiological assessments were done in all patients and included pure tone and speech audiometry, tympanometry, and evoked response audiometry (ERA). Short increment sensitivity index (SISI) and tone decay tests were also performed. On audiometry, the pure tone average (PTA) was calculated at the frequencies 500, 1000, 2000 and $3000 \mathrm{~Hz}$, and the speech reception threshold (SRT) and speech discrimination score (SDS) were also calculated. The ERA (Intelligent Hearing System, Multichannel Evoked Potential System, Miami, FL, USA) parameters were: filters 100-3000 Hz, repetitions 1024, stimulus intensity $80 \mathrm{~dB} \mathrm{HL}$, and stimulation rate 11 rarefaction clicks per second. On ERA testing, the wave V/I ratio, which should be above 1 , was calculated to evaluate the wave morphology. A ratio below 1 was considered as abnormal morphology.

\section{Statistics}

Statistical analysis was performed with Statistical Package for the Social Sciences for Windows (Release 8.0, SPSS/PC+ Inc, Chicago, IL, USA). One way analysis of variance (ANOVA) was used to compare group 1, group 2, and controls. Homogeneity of variance was tested. Post hoc tests were applied, and Tukey test results were taken into consideration for equal variances and Tamhane results for unequal variances. Dunnett results were used for comparisons with control subjects. The paired $t$ test was applied to compare the right and left ear. The Mann-Whitney U test was used for confirmation of the results as indicated. The results were expressed as mean (SD).

\section{RESULTS}

In group 1, there were nine patients (seven men and two women) with a mean age of 33.9 years (range 22-57). In group 2, there were eight patients (six men and two women) with a mean age of 34.4 years (range 20-51). The age and sex distribution between the groups were comparable (ANOVA, $\mathrm{p}>0.05)$. All Behçet's disease patients had oral ulcerations $(100 \%)$. Sixteen patients $(94.1 \%)$ had skin lesions (pseudofolliculitis, acneiform lesions, thrombophlebitis, erythema nodosum) and genital ulcerations were present in 15 (88.2\%). The pathergy test positivity (the observation of a pustular reaction by a physician at $24-48$ hours after a needlestick to the forearm) was present in nine patients (52.9\%). Eight of 17 patients (47.0\%; group 2) had bilateral ocular type of Behçet's disease ${ }^{25}$ with end stage manifestations (phthisical or completely blind eye). Additional findings were arthralgia in $14(82.3 \%)$, migraine-like headache in seven $(41.1 \%)$, and appendicitis-like pain or melaena in five $(29.4 \%)$.

The otoscopic examination was normal in all patients in group 1 while some sort of abnormalities were detected in group 2, which included atrophic tympanic membrane in two, myringitis in one, myringosclerosis in one, and dermatitis in the external auditory canal in four patients. Dix-Halpike positional testing was normal and did not induce nystagmus or a falling sensation. The PTA, SRT, and SDS were not significantly different between the right and left ears of the patients (paired $t$ test, $\mathrm{p}>0.05$ ) as well as between group 1 and group 2 (ANOVA, $\mathrm{p}>0.05$ ) (table 1 ). Likewise, the frequency specific comparison of the pure tone results of the right and left ear was not significantly different (paired $t$ test, $\mathrm{p}>0.05$ ) (table 2). The pure tone results of the Behçet patients and healthy controls were significantly different at the frequencies $4000 \mathrm{~Hz}(\mathrm{f}=3.950, \mathrm{df}=2, \mathrm{p}=0.03)$ and $6000 \mathrm{~Hz}(\mathrm{f}=4.307, \mathrm{df}=2, \mathrm{p}=0.02)$. In all patients and controls, the tympanometry was type A, SISI was ambiguous as to site, and tone decay was normal.

The types of the audiograms obtained in group 1 were flat, notched, and sloping in $72.2 \%, 16.7 \%$ and $11.1 \%$ of the patients, respectively. In group 2, the audiograms were flat, sloping, and rising in $43.8 \%, 37.5 \%$ and $18.8 \%$, respectively. In general, a sloping audiogram with bilateral symmetric and mild sensorineural hearing loss was obtained for both group 1 and group 2 (fig 1 ).

Table 2 Frequency specific pure tone results in patients without (group 1) or with (group 2) end stage ocular involvement and healthy controls; results are in $\mathrm{dB}$

\begin{tabular}{|c|c|c|c|c|c|c|c|c|}
\hline \multirow[b]{3}{*}{ Frequency (Hz) } & & & \multicolumn{4}{|c|}{ End stage ocular involvement } & & \\
\hline & \multicolumn{2}{|c|}{ Overall $(n=17)$} & \multicolumn{2}{|c|}{ Group 1 (n=9) } & \multicolumn{2}{|c|}{ Group $2(n=8)$} & \multicolumn{2}{|c|}{ Controls $(n=13$ ) } \\
\hline & Right & Left & Right & Left & Right & Left & Right & Left \\
\hline 250 & 18.5 & 18.5 & 16.7 & 16.7 & 20.6 & 20.6 & 10.1 & 10.5 \\
\hline 500 & 14.4 & 16.2 & 12.2 & 12.8 & 16.9 & 20 & 10.6 & 10.5 \\
\hline 1000 & 12.6 & 13.5 & 11.7 & 10 & 13.8 & 17.5 & 12.4 & 12.4 \\
\hline 2000 & 15 & 17.1 & 12.2 & 15 & 18.1 & 19.4 & 9.1 & 9.9 \\
\hline 4000 & 24.7 & 29.1 & 22.8 & 27.8 & 26.9 & 30.6 & 12.8 & $13.7^{*}$ \\
\hline 6000 & 30.6 & 34.7 & 25.6 & 32.2 & 36.3 & 37.5 & 14.2 & $13.7^{*}$ \\
\hline
\end{tabular}


Table 3 Evoked response audiometry results in patients without (group 1) or with (group 2) end stage ocular involvement and healthy controls

\begin{tabular}{|c|c|c|c|c|c|c|c|c|c|}
\hline \multirow[b]{2}{*}{ Ocular involvement } & \multicolumn{3}{|c|}{ Absolute latency (ms) } & \multicolumn{3}{|c|}{ Interpeak latency (ms) } & \multicolumn{3}{|c|}{ Wave amplitudes $(\mu v)$} \\
\hline & 1 & III & V & I-III & I-V & III-V & 1 & III & V \\
\hline Group 1 (mean) & 1.35 & 3.49 & 5.47 & $2.25^{*}$ & 4.13 & 1.99 & 0.16 & 0.22 & 0.23 \\
\hline SD & 0.42 & 0.63 & 0.71 & 0.39 & 0.47 & 0.42 & 0.11 & 0.12 & 0.10 \\
\hline Group 2 (mean) & 1.23 & 3.15 & 5.13 & 1.91 & 3.89 & 2.02 & 0.18 & 0.23 & 0.25 \\
\hline SD & 0.18 & 0.32 & 0.16 & 0.33 & 0.21 & 0.35 & 0.11 & 0.13 & 0.14 \\
\hline Controls (mean) & 1.25 & 3.1 & 5.17 & 1.88 & 3.9 & 2.11 & 0.19 & 0.21 & 0.27 \\
\hline SD & 0.31 & 0.4 & 0.27 & 0.4 & 0.39 & 0.23 & 0.1 & 0.14 & 0.22 \\
\hline
\end{tabular}

From their history, five (29.4\%), seven (41.1\%), two $(11.8 \%)$, and one $(5.9 \%)$ of the patients were complaining of hearing loss, tinnitus, vertigo and dizziness, respectively. Of five patients who complained of hearing loss, three were in group 1 and two were in group 2 . When the frequency specific pure tone audiometry results of the patients who complained of hearing loss were compared to those who did not complain, there was no statistically significant difference (ANOVA, $p>0.05$ ). However, a statistically significant difference was found between their I-III interpeak intervals on ERA testing $(\mathrm{z}=-2.132, \mathrm{df}=2, \mathrm{p}=0.03)$, which were 2.5 (0.43) and 1.8 (1.5) msec, respectively. Their SDSs, which were also significantly different $(\mathrm{z}=-3.312, \mathrm{df}=2, \mathrm{p}<0.01)$, were $84.4 \%$ and $93.7 \%$, respectively. The acoustic reflex arc was normal in all except three patients, of whom two had bilateral and one had unilateral absence of the reflex at $4000 \mathrm{~Hz}$. These three patients were among those who complained of hearing loss as well.

According to ERA results the absolute and interpeak latencies, and the amplitudes of the waves, were similar in both groups except for interpeak interval I-III, which was significantly longer in group 1 than in group 2 and controls $(\mathrm{f}=14.535, \mathrm{df}=2, \mathrm{p}<0.01)$ (table 3$)$. An abnormal wave morphology was present only in four $(23.5 \%)$ of 17 patients, and all were in group 1.

There was no relationship between the presence or absence of end stage ocular involvement and the otological parameters studied (ANOVA, $\mathrm{p}>0.05$ ) excluding I-III interval on ERA. The mean duration of disease was 5.1 and 13.1 years in group 1 and group 2, respectively, and there was a relationship between the end stage ocular involvement and the duration of disease $(f=6.895, \mathrm{df}=2, \mathrm{p}=0.02$ ). There was no relationship between the otological symptoms or audiological test results and the duration of Behçet's disease (ANOVA, $\mathrm{p}>0.05)$.

\section{DISCUSSION}

As the underlying pathology in Behçet's disease is a chronic and relapsing vasculitis, it can involve a variety of organs and

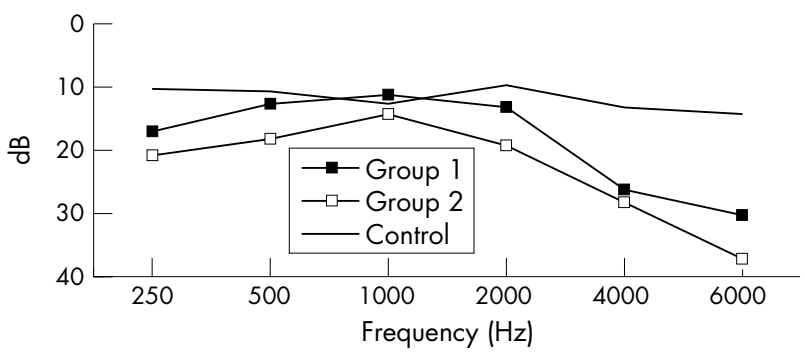

Figure 1 Representative audiograms of the patients with Behçet's disease without (group 1) and with (group 2) with end stage ocular involvement and healthy controls. systems. Vascular and autoimmune aetiology can also be associated with neurotological and ocular manifestations. In Behçet's disease, the vasculitis that can affect all systems in the body, which may possibly impact on both ophthalmological and neurotological status. Therefore, presence of a correlation between ocular and otological involvement might be possible in Behçet's disease.

It has been suggested that inner ear involvement is a late complication, appearing almost a decade after the initial manifestations. ${ }^{17}$ However, reports on the correlation between the inner ear involvement and the duration of Behçet's disease are contradictory. ${ }^{17} 26$ In our study, there was no relationship between otological symptoms or audiological test results, and the duration of Behçet's disease. Although there was no relationship between the severity of ocular involvement and neurotological status, otoscopy was normal in group 1 patients while there were some abnormalities in group 2 . These otoscopically detected abnormalities were observed in the tympanic membrane (atrophy, myringitis, sclerosis) and the skin of the external auditory (dermatitis). These can be considered the main otoscopic findings in Behçet's disease that are likely to occur through dermatitis.

In Behçet's disease, auditory and vestibular abnormalities were found in $73 \%$ and $54 \%$ of the patients, respectively. ${ }^{28}$ According to another study, hearing disturbances and vertigo were the complaints in $62 \%$ and $37 \%$, respectively. ${ }^{17}$ According to our results, $11.8 \%$ and $5.9 \%$ of the patients complained of vertigo and dizziness, respectively. It is apparent that cochlear involvement is more common than vestibular involvement. The different rates in the literature might have resulted from the various parameters included in the estimation of the cochlear and vestibular involvement, and lack of standardisation.

Dix-Halpike positional testing was normal and did not induce nystagmus. Further investigation of the vestibular functions was not performed in the patients who described vertigo and dizziness, because these occasional symptoms were not persistent or incapacitating. There was no significant correlation between the presence of vestibular symptoms and ocular involvement. That is, the vestibular symptoms are subtle even in the presence of end stage ocular involvement.

It has been suggested that there is no clear typical pattern of hearing loss for Behçet's patients. ${ }^{21}$ This statement is true when we evaluate the audiograms of the patients individually. However, when we draw the audiogram based on the frequency specific PTA, we find the typical audiogram for the patients. Accordingly, the main audiogram obtained in both groups was a sloping audiogram showing bilateral symmetric and mild sensorineural hearing loss. However, an audiogram of this kind should not be considered diagnostic for the otological involvement in Behçet's disease, because it can be seen in some other conditions like presbycusis. 
The PTA, SRT, and SDS were not significantly different between the right and left ears of the patients as well as between the group 1 and group 2. Likewise, the frequency specific comparison of the pure tone results was not significantly different. The symmetricity of the hearing loss in both ears is suggestive of the impact of systemic disease, that is Behçet's disease, and possibly through autoimmune vasculitis.

The middle ear and eustachian tube functions were spared as there was no conductive hearing loss and the tympanogram was normal. High SISI scores have been reported in some patients with Behçet's disease. ${ }^{21} 27$ The SISI scores of all patients in our series were not diagnostic for the site of lesion-that is, whether it was cochlear or retrocochlear. Although the normal tone decay result may suggest absence of retrocochlear involvement, a prolonged I-III interval on ERA indicates an involvement in the conduction between the cochlear nerve and brainstem neural integrators.

According to one study, the peripheral involvement is more common than central involvement for both auditory and vestibular lesions, and there is no correlation between the audiovestibular lesions and other organ lesions, disease duration, or age or sex of the patients..$^{28}$ According to another study, the visual impairment that occurred in some patients did not correlate with the vestibulo-ocular reflex elevation, which might be due to the presence of a latent brainstem abnormality. ${ }^{29}$ It was also suggested that there might be a latent brainstem lesion, which may manifest by vestibulopathy. ${ }^{22}$ According to our results, there was both peripheral and central involvement, the latter being more common and severe. However, statistical analyses showed the absence of an association between ear involvement and the severity of the ocular involvement.

The cochlear symptoms were more prevalent than the vestibular symptoms in Behçet's disease. Seven (41.1\%) patients had tinnitus and five $(29.4 \%)$ had hearing loss. Presence or absence of cochlear symptoms was not associated with ocular involvement. When the frequency specific PTAs of the patients who complained of hearing loss were compared to those who did not complain of hearing loss, there was no significant difference. The acoustic reflex arc was normal in all except three patients, of whom two had bilateral and one had unilateral absence at $4000 \mathrm{~Hz}$. These three patients were among those who also complained of hearing loss. Therefore, the presence of acoustic reflex abnormality may suggest inner ear involvement in the disease. The $4000 \mathrm{~Hz}$ frequency corresponds to the basal turn of the cochlea. High frequency hearing loss indicates damage in the basal turn of the cochlea. A lesion in the cochlea as the cause of sensorineural deafness has been suggested previously. ${ }^{22}$ However, the interpeak intervals of wave I-III and SDS were found to be significantly longer when the patients complained of hearing loss. These ERA results and SDSs suggest that there is a retrocochlear pathology, particularly in the cochlear nerve and lower brainstem that makes the patient complain of hearing loss. The possibility of a bilateral central lesion of the eight cranial nerves should be considered in Behçet's disease. ${ }^{19}$ Abnormal evoked potential may also provide sensitive information, which may show the presence of subclinical lesions in the central nervous system.

A number of ERA abnormalities can be seen in Behçet's disease. In a patient who had mild to moderate sensorineural hearing loss, the interpeak intervals were found to be normal on ERA testing. ${ }^{30}$ In another series, elongation in the absolute latency of wave I was the only abnormality noted in two of 25 patients. ${ }^{28}$ It was proposed that ERA was abnormal in $52 \%$ of the patients with neurological manifestations and in $31 \%$ without, because of decreased amplitude of wave V, prolonged I-III or III-V interpeak latencies, or uncertain/ absent waves III and/or V. ${ }^{31}$ In another study, $64.7 \%$ of the patients showed an absence of wave I, III or V, or a prolongation of the interpeak latency I-III or III-V. ${ }^{32}$ In our series, the main abnormality was in the I-III interpeak interval and in the wave morphology. The absolute and interpeak latencies, and the amplitudes of the waves, were similar in both groups except for interpeak interval I-III, which was significantly longer in group 1 than group 2 . Abnormal wave morphology was present only in $23.5 \%$ of the patients, and all were in group 1 . These data suggest a brainstem involvement in Behçet's disease. In addition to that, there were more abnormalities on ERA testing in the absence of ocular involvement (group 1), though we expected to find more ERA abnormalities in group 2 in which the disease was considered severe as far as the end stage ocular involvement was concerned. These results also indicate that even if there was retrocochlear involvement in Behçet's disease, its severity would not depend on the severity of the ocular involvement.

In conclusion, otological symptoms or audiological test results did not correlate with the duration of or ocular involvement in Behçet's disease. However, there was a relationship between ocular involvement and duration of disease. This may suggest that the severity of ocular involvement in Behçet's disease is a function of time. However, neurotological involvement is not time dependent. Although the main underlying pathogenetic factor in Behçet's disease is the autoimmune vasculitis, the mechanisms involved in the pathogenesis of neurotological and ocular damage may be different. Alternatively, the differential involvement of certain organ systems may not reflect the nature of the disease process itself, but rather the manner in which each organ responds to injury.

\section{Authors' affiliations}

Y A Bayazit, Department of Otolaryngology, Faculty of Medicine, Gazi University, Besevler, Ankara, Turkey

C Evereklioğlu, Department of Ophthalmology, Faculty of Medicine, Erciyes University, Kayseri, Turkey

E Ozer, M Kanlikama, Department of Otolaryngology, Faculty of Medicine, Gaziantep University, Gaziantep, Turkey

N Kırtak, S İnalöz, Department of Dermatology, Faculty of Medicine, Gaziantep University, Gaziantep, Turkey

N Bayazit, Department of Clinical Microbiology and Infectious Diseases, Faculty of Medicine, Gaziantep University, Gaziantep, Turkey

When this study was conduced, the first author was working in Department of Otolaryngology, Faculty of Medicine, Gaziantep University, Gaziantep, Turkey, and the second author was working in Department of Ophthalmology, Faculty of Medicine, Erciyes University, Kayseri, Turkey.

\section{REFERENCES}

1 Behçet H. Über rezidivierende Aphthöse, durch ein Virus verursachte Geschwüre am Mund, am Auge und an den Genitalien. Dermatol Wochenscrhr 1937;105:1152-7.

2 Onder M, Gurer MA. The multiple faces of Behçet's disease and its aetiological factors. J Eur Acad Dermatol Venereol 2000;15:126-36.

3 Ghate JV, Jorizzo JL. Behçet's disease and complex aphthosis. J Am Acad Dermatol 1999;40:1-18.

4 Mizuki N, Ohno S, Kamata K, et al. Immunogenetic mechanism of Behçet's disease. Nippon Ganka Gakkai Zasshi 1991;95:783-9.

5 Sakane T, Takeno M, Suzuki N, et al. Behçet's disease. N Engl J Med 1999;341:1284-91

6 Evereklioglu C, Er H. Increased corneal thickness in active Behçet's disease. Eur J Ophthalmol 2002;12:24-9.

7 Evereklioglu C, Ozbek E, Er H, et al. Urinary adrenomedullin levels are increased and correlated with plasma concentrations in patients with Behçet's syndrome. Int J Urol 2002;9:296-303.

8 Gunen H, Evereklioglu C, Kosar F, et al. Thoracic involvement in Behçet's disease and its correlation with multiple parameters. Lung 2000;178:161-70.

9 Everklioglu C, Yurekli M, Er H, et al. Increased plasma adrenomedullin levels in patients with Behçet's disease. Dermatology 2000;201:312-5. 
10 Everklioglu $\mathrm{C}$, Turkoz $\mathrm{Y}, \mathrm{Er} \mathrm{H}$, et al. Increased nitric oxide production in patients with Behçet's disease: is it a new activity marker? J Am Acad Dermatol 2002;46:50-4

11 Evereklioglu C, Er H, Turkoz Y, et al. Serum levels of TNF- $\alpha$, sIL-2R, IL-6, and IL-8 are increased and associated with elevated lipid peroxidation in patients with Behçet's disease. Mediators of Inflammation 2002;1 1:87-93.

12 Turkoz Y, Evereklioglu C, Mehmet N, et al. Plasma malondialdehyde levels in Behçet's disease with or without ocular involvement. Advances in Reproduction 2000;4:31-6.

13 Lakhanpal S, Tani K, Lie JT, et al. Pathologic features of Behçet's syndrome: a review of Japanese autopsy registry data. Hum Pathol 1985; 16:790-5.

14 Gripshover BM, Ellner JJ. Chronic meningitis. In: Mandell GL, eds. Principles and practice of infectious diseases. 4th Ed. Edinburgh: Churchill Livingstone, 1995:865-974.

15 Alajouanine T, Castaigne V, Lhermitte F, et al. La meningo-encephalite de la maladie de Behçet. Presse Med 1961;69:2579-82.

16 Kawakita H, Nishimura M, Satoh Y, et al. Neurological aspects of Behçet's disease. A case report and clinico-pathological review of the literature in Japan. J Neurol Sci 1967;5:417-39.

17 Brama I, Fainaru M. Inner ear involvement in Behçet's disease. Arch Otolaryngol 1980;106:215-7.

18 Okamoto M, Shitara T, Nishihata S, et al. Sensorineural hearing loss in patients with Behcet's disease. J Otolaryngol Jpn 1982;85:667-73.

19 Belkahia A, Ben Ayed H, Ben H'mida M, et al. Auditory and vestibular lesions in Behçet's disease. Ann Otolaryngol Chir Cervicofac 1982;99:469-76.

20 Andreoli C, Savastano M. Audiologic pathology in Behçet's syndrome. Am J Otol 1989;10:466-7.
21 Elidan J, Levi $\mathrm{H}$, Cohen $\mathrm{E}$, et al. Effect of cyclosporin $\mathrm{A}$ on the hearing loss in Behçet's disease. Ann Otol Rhinol Laryngol 1991;100:464-8.

22 Gemignani G, Berrettini S, Bruschini P, et al. Hearing and vestibular disturbances in Behçet's syndrome. Ann Otol Rhinol Laryngol $1991 ; 100: 459-63$

23 International Study Group for Behçet's disease. Criteria for diagnosis of Behçet's disease. Lancet 1990;335:1078-80.

24 Bloch-Michel E, Nussenblatt RB. International Uveitis Study Group recommendations for the evaluation of intraocular inflammatory disease. Am J Ophthalmol 1987;103:234-5.

25 BenEzra D, Maftzir G, Kalichman I, et al. Serum levels of interleukin-2 receptor in ocular Behçet's disease. Am J Ophthalmol 1993;115:26-30.

26 Soylu L, Aydogan B, Soylu M, et al. Hearing loss in Behçet's disease. Ann Otol Rhinol Laryngol 1995;104:864-7.

27 Everklioglu C, Cokkeser Y, Doganay S, et al. Audio-vestibular evaluation in patients with Behçet's disease. J Laryngol Otol 2001;115:704-8.

28 Pollak L, Luxon LM, Haskard DO. Labyrinthine involvement in Behçet's syndrome. J Laryngol Otol 2001;115:522-9.

29 Sugasawa J, Ishikawa S. Vestibulo-ocular reflex abnormality in Behçet's disease. Jpn J Ophthalmol 1986;30:91-9.

30 Smith LN. Unilateral sensorineural hearing loss in Behçet's disease. Am J Otolaryngol 1994;15:286-8.

31 Stigsby B, Bohlega S, al-Kawi MZ, et al. Evoked potential findings in Behçet's disease. Brain-stem auditory, visual, and somatosensory evoked potentials in 44 patients. Electroencephalogr Clin Neurophysiol 1994;92:273-81.

32 Nakamura $Y$, Takahashi $M$, Kitaguchi $M$, et al. Brainstem auditory and somatosensory evoked potentials in neuro-Behçet's syndrome. Jpn J Psychiatry Neurol 1989;43:191-200.

FILLER

\section{Putting exams through their PACES} ical examinations are now objective and structured with visitations to multiple stations where all candidates have to perform the same tasks while examiners look on. Apart from history taking and communication skills stations there is minimal opportunity to relate to candidates. I find the "non-talking" stations boring and hardly ever memorable.

In contrast the old style exams often provided memorable incidents. The candidate gave a full history of her patient's respiratory problems but unfortunately had not realised that he kept racing pigeons. My fellow examiner threw her a verbal lifeline "If I were to tell you he kept racing pigeons would this affect management?" The candidate was visibly grateful. "I would tell him to send them away". My colleague found himself severing her lifeline as he said "but racing pigeons are homing pigeons".

Memorable interactions are rarer with station based exams, but do occur in the "talking" stations. The rules were clear. Each candidate had 14 minutes talking to a simulated patient, one minute for reflection, and five minutes discussion with the examiners. I had written a communication skills scenario which involved a forceful businessman who had to be persuaded his diabetes required attention. His scripted opening lines were " $I$ ' $m$ a busy man doctor and diabetes seems simple. Tell me about it. And how long is this consultation going to be as I have an important meeting". The candidate gave a wry smile and replied "Exactly 14 minutes".

P D Welsby

Western General Hospital, Edinburgh EH4 2XJ, UK; p.welsby@ed.ac.uk 